\title{
Magnetic Properties of Polymers Containing Tetraphenyl- porphinatosilver(II) as Their Side Chains
}

\author{
Xian Su Cheng, Mikiharu KamaChI, Wasuke Mori, ${ }^{*}$ \\ and Michihiko KISHITA* \\ Department of Macromolecular Science, Faculty of Science, \\ Osaka University, Toyonaka, Osaka 560, Japan \\ * Department of Chemistry, College of General Education, \\ Osaka University, Toyonaka, Osaka 560, Japan
}

(Received July 3, 1989)

\begin{abstract}
The magnetic properties of polymers containing tetraphenylporphinatosilver(II) (TPPAg(II)) were investigated by measurement of magnetic susceptibility and ESR spectra and a monomer model. The results reveal that poly[5-(4-acryloyloxyphenyl)-10,15,20-triphenylporphinatosilver(II)] (polyAOTPPAg(II)) has much stronger antiferromagnetic interaction between TPP$\mathrm{Ag}$ (II) moieties than the monomer models, which indicates that the polymer chain plays an important role for the occurrence of magnetic interaction. A comparison of the magnetic properties of polyAOTPPPAg(II) with that of poly[5-(4-methacryloyloxyphenyl)-10,15,20-triphenylporphinatosilver(II)] (polyMAOTPPAg(II) showed that the relative location of TPPAg(II) moieties is an important factor for the magnetic ordering. Moreover, the increase in the distance between TPPAg(II) moieties due to MMA units in the copolymers decreased the magnetic interaction. These findings are consistent with the previous concept that the antiferromagnetic exchange interaction in polyAOTPPAg(II) is possibly caused by super-exchange interactions through the $\mathrm{C}=\mathrm{O}$ group between $\mathrm{Ag}(\mathrm{II})$ ions.
\end{abstract}

KEY WORDS Polymer / Magnetic Property / Magnetic Susceptibility / ESR / Antiferromagnetic Interaction / Tetraphenylporphyrin / Tetraphynylprophinatosilver(II) / Poly[5-(4-methacryloyloxyphenyl)10,15, 20-triphenylporphinatosilver(II)] /

Polymers containing paramagnetic species in their side chains are expected to serve as new magnetic materials, because a magnetic ordering of the paramagnetic species through an exchange interaction of unpaired electrons is possible in the polymers. ${ }^{1-3}$ Kamachi et al..$^{4.5}$ measured the magnetic susceptibility of polymers containing piperidinyloxyl and verdazyl in their side chains, and compared their magnetic behaviors with those of the corresponding monomers. The polymer effect on the magnetic ordering was found in these systems, but the exchange interactions between these organic radicals were too weak for the polymers to be used as a magnetic material. Eaton et al. ${ }^{6}$ reported a ESR study of spin- labeled silver porphyrins and showed that the silvernitroxyl exchange interaction was 1000 gauss. Kamachi et al. undertook the preparation of copolymers containing TPPAg(II) or TPPCu(II) and nitroxyl in their side chains. Before preparing the copolymer, Kamachi et al. $^{3}$ synthesized poly[5-(4-acryloyloxyphenyl)-10,15,20-triphenylporphinatosilver(II)] (polyAOTPPAg(II)) in their side chains and found that the polymer was strongly antiferromagnetic. They suggested that the origin of the strong antiferromagnetic interaction of the polymer is superexchange interactions between TPPAg(II) moieties in their side chains through the carbonyl oxygen. The superexchange interaction have not yet been checked 


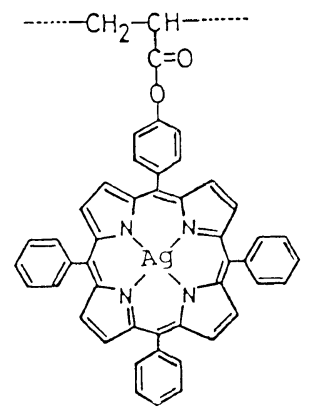

PolyAOTPPAg(II)

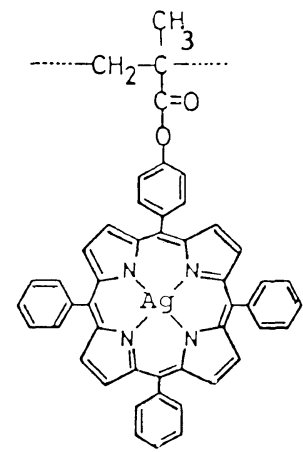

PolyMAOTPPAg(II) directly. For an understanding of the origin of the magnetic interaction of the polymer containing tetraphenylporphinatosilver(II), we investigated the magnetic behavior by the following two indirect methods: one of them is measurement of the magnetic properties of poly[5-(4-methacryloyloxyphenyl)-10,15,20triphenylporphinatosilver(II)] (polyMAOTPPAg(II)), which is considered to have a stable conformation different from polyAOTPP$\mathrm{Ag}$ (II) owing to methyl groups bound to the main chain. The other is the measurement of the magnetic properties of copolymers of AOTPPAg(II) and MMA, in which the distance between tetraphenylporphinatosilver(II) moieties was controlled by changing the ratio of AOTPPAg(II) to MMA.

This paper describes the magnetic properties of polyMAOTPPAg(II) and poly[AOTPPAg(II)-co-MMA]s with different compositions along with their monomer model in detail.

\section{EXPERIMENTAL}

\section{Materials}

5-(4-Hydroxyphenyl)-10,15,20-triphenylporphyrin (HOTPP) was prepared from benzaldehyde, $p$-hydroxybenzaldehyde and pyrrole according to the method of Little et al. ${ }^{7}$ Yield $4.9 \%$.

Syntheses of poly[5-4-acryloyloxyphenyl)10,15,20-triphenylporphyrin] (polyAOTPP) and poly[5-(4-methacryloyloxyphenyl)-10, 15,-20-triphenylporphyrin] (polyMAOTPP) were described previously. ${ }^{8,9}$

Poly[5-(4-acryloyloxyphenyl)-10,15,20-triphenylporphyrin-co-methyl methacrylate] [PolyAOTPP-co-MMA] was synthesized by the copolymerization of AOTPP with MMA. The copolymerization was performed at $60^{\circ} \mathrm{C}$ in benzonitrile using AIBN as an initiator. The copolymer was isolated by precipitation in methanol and purified by three dissolutionprecipitation-separation steps from THFmethanol. The monomer molar ratio of AOTPP to MMA was $1: 1$ and $1: 3$. The copolymer compositions, which were estimated by elemental analysis of nitrogen content, were $1: 0.8$ and $1: 2.6$. Anal. Calcd. for poly[AOTPP-co-MMA] [AOTPP: $\mathrm{MMA}=1: 0.8$ ] $\left(\mathrm{C}_{47} \mathrm{H}_{32} \mathrm{~N}_{4} \mathrm{O}_{2}\right)_{1}:\left(\mathrm{C}_{5} \mathrm{H}_{8} \mathrm{O}_{2}\right) 0.8: \mathrm{C}, 80.08 \% ; \mathrm{H}$, $5.06 \%$; N, $7.32 \%$. Found: C, $80.12 \%$; $5.10 \%$; N, $7.28 \%$. Anal. Calcd. for poly[AOTPP-co-MMA] [AOTPP : $\mathrm{MMA}=1: 2.6$ ] $\left(\mathrm{C}_{47} \mathrm{H}_{32} \mathrm{~N}_{4} \mathrm{O}_{2}\right)_{1}:\left(\mathrm{C}_{5} \mathrm{H}_{8} \mathrm{O}_{2}\right)_{2.6}: \mathrm{C}, 75.25 \% ; \mathrm{H}$, $5.63 \%$; N, $5.93 \%$. Found: C, $75.81 \%$; $5.66 \%$; N, $5.91 \%$.

Poly[5-(4-acryloyloxyphenyl)-10,15,20-triphenylporphyinatosilver(II)] [PolyAOTPPAg(II)] was prepared as follows:

PolyAOTPP $(0.21 \mathrm{~g})$ and silver acetate $(0.71 \mathrm{~g})$ were dissolved in $200 \mathrm{ml}$ of tetrahydrofuran. The solution was refluxed for $9 \mathrm{~h}$ and the solvent was removed by distillation. Chloroform was added to the residue to dissolve the polymer and the insoluble part was 
filtered. The filtrate was washed with water to remove silver acetate, and the polymer was isolated by evaporating chloroform. The obtained polymer was dried in vacuum, dissolv ed in benzene, and freeze-dried. Yield $0.22 \mathrm{~g}$ $(88 \%)$. The content of porphinatosilver(II) in the porphyrin moieties was $90 \%$ by atomic absorption spectroscopy. Anal. Calcd. for polyAOTPPAg(II) $\quad\left(\mathrm{C}_{47} \mathrm{H}_{30} \mathrm{~N}_{4} \mathrm{O}_{2} \mathrm{Ag}\right): \mathrm{C}$, $71.40 \%, \mathrm{H}, 3.82 \%, \mathrm{~N}, 7.09 \%, \mathrm{Ag}, 13.6 \%$. Found: C, $71.66 \% ; \mathrm{H}, 4.67 \%$; N, 6.14; Ag, $12.2 \%$.

PolyMAOTPPAg(II) and poly[AOTPPAg(II)-co-MMA] were prepared by the same method as polyAOTPPAg(II), except that the refluxing time was $15 \mathrm{~h}$ and that the amount of refluxing solvent was increased $30 \%$. By these improvements, the silver contents in the porphyrin moieties of both polymers increased to $96 \%, 95 \%$ and $94 \%$, respectively. Anal. Calcd. for polyMAOTPPAg(II) $\left(\mathrm{C}_{48} \mathrm{H}_{32} \mathrm{~N}_{4} \mathrm{O}_{2} \mathrm{Ag}\right)$ : $\mathrm{C}, 71.65 \%$; H, $4.01 \%$; N, 6.96\%; Ag, $13.4 \%$. C, $71.14 \% ; \mathrm{H}, 4.53 \%$; N $6.60 \%$ Ag, 12.9 $\%$. Anal. Calcd. for poly[AOTPPAg(II)-coMMA] [AOTPPAg(II): MMA] $=1: 0.8$ ] $\left(\mathrm{C}_{47^{-}}\right.$ $\left.\mathrm{H}_{30} \mathrm{~N}_{4} \mathrm{O}_{2} \mathrm{Ag}\right)_{1}:\left(\mathrm{C}_{5} \mathrm{H}_{8} \mathrm{O}_{2}\right)_{0.8}: \mathrm{C}, 70.35 \% ; \mathrm{H}$, $4.21 \%$; N $6.43 \%$; Ag, $12.4 \%$. Found: C, $70.02 \%$; H, $4.64 \%$; N, $6.32 \%$, Ag, $11.5 \%$. Anal. Calcd. for poly[AOTPPAg(II)-co-MMA] [AOTPPAg(II) : MMA = $1: 2.6]\left(\mathrm{C}_{47} \mathrm{H}_{30} \mathrm{~N}_{4} \mathrm{O}_{2}\right.$ $\mathrm{Ag})_{1}:\left(\mathrm{C}_{5} \mathrm{H}_{8} \mathrm{O}_{2}\right)_{2.6}: \mathrm{C}, 68.57 \% ; \mathrm{H}, 4.87 \%$, N, $5.53 \%$; Ag, $10.3 \%$. Found. C, $67.43 \%$; $4.97 \%$; N, $5.31 \%$; Ag, $9.9 \%$.

5-(4-Propyloyloxyphenyl)-10,15,20-triphenylporphyrin (POTPP) was synthesized as follows: HOTPP (1.51 g) and triethylamine $(0.84 \mathrm{~g})$ were dissolved in $200 \mathrm{ml}$ of tetrahydrofuran. To this solution was added dropwise $0.80 \mathrm{~g}$ of propionyl chloride with stirring in $20 \mathrm{~min}$; then the reaction mixture was stirred at room temperature for $3 \mathrm{~h}$. Removal of the solvent afforded a crude product, which was then dissolved in chloroform and chromatographed on a silica gel column using chloroform as an eluent. POTPP was isolated by removal of the solvent from the first fraction and dried at $120^{\circ} \mathrm{C}$ for $12 \mathrm{~h}$ under reduced pressure. Yield, $1.48 \mathrm{~g}(90 \%)$. IR (KBr): $1775 \mathrm{~cm}^{-1}\left(v_{\mathrm{C}=\mathrm{O}}\right)$. Anal. Calcd. for POTPP $\left(\mathrm{C}_{47} \mathrm{H}_{34} \mathrm{~N}_{4} \mathrm{O}_{2}\right)$ : C, $82.19 \%$; $\mathrm{H}, 4.99 \%$, $\mathrm{N}, 8.16 \%$. Found: C, $82.07 \%$;, $5.04 \%$, $8.13 \%$.

5-(4-Propyloyloxyphenyl)-10,15,20-triphenylporphinatosilver(II) (POTPPAg(II)) was prepared according to Dorough et al. ${ }^{10}$ Yield $95 \%$. The content of porphinatosilver(II) in porphyrin moieties was almost $100 \%$. Anal. Calcd. for POTPPAg(II) $\left(\mathrm{C}_{47} \mathrm{H}_{33} \mathrm{~N}_{4} \mathrm{O}_{2} \mathrm{Ag}\right)$ : C, $72.27 \%$; $\mathrm{H}, 4.07 \%$; N $7.07 \%$; Ag, $13.6 \%$. Found. C, $71.02 \%$; H, $4.36 \%$; N $6.95 \%$; Ag, $13.5 \%$.

\section{Measurement}

ESR spectra were recorded with a JEOL Model JESFE IX ESR spectrometer with a $100 \mathrm{kHz}$ modulator. $g$-values were estimated using $\mathrm{MnO}$ as the standard sample.

Gram magnetic susceptibility $\left(\chi_{\mathrm{g}}\right)$ was determined by the Gouy method at room temperature using distilled water $\left(\chi_{\mathrm{g}}=-0.72 \times\right.$ $10^{-6}$ cgsemu) as the standard. The contribution of atmosphere to the magnetic susceptibility was corrected by adding $0.03 \times 10^{-6}$ cgsemu to $\chi_{\mathrm{g}}$. The temperature dependence of $\chi_{\mathrm{g}}$ was determined by the Faraday method, using a Cahn 1000 electric balance in a temperature range from $4 \mathrm{~K}$ to $300 \mathrm{~K}$. The molar magnetic susceptibility $\left(\chi_{\mathrm{M}}=\chi_{\mathrm{g}} \times M\right.$; $M$ is molecular weight of TPPAg(II) or monomer unit of the polymer) was corrected for diamagnetic contribution from the ligands. The $\chi_{\text {dia }}$ values for TPP, POTPP, polyAOTPP and polyMAOTPP were estimated as $-535 \times$ $10^{-6},-560 \times 10^{-6},-564 \times 10^{-6}$, and $-575 \times$ $10^{-6}$ cgsemu, respectively, by direct measurement. The effective magnetic moment $\left(\mu_{\text {eff }}\right)$ was calculated by the following equation: ${ }^{11}$

$$
\mu_{\text {eff }}=2.83 \sqrt{X_{\mathrm{M}} \cdot T}
$$




\section{RESULTS AND DISCUSSION}

\section{Magnetic Susceptibility}

The gram magnetic susceptibilities and effective magnetic moments of TPPAg(II), POTPPAg(II), polyAOTPPAg(II), and polyMAOTPPAg(II) at room temperature are listed in Table I. The values of $\chi_{\mathrm{g}}$ and $\mu_{\mathrm{eff}}$ of polyAOTPPAg(II) at room temperature are considerably smaller than those of TPPAg(II), POTPPAg(II), and polyMAOTPPAg(II).

Previously, ${ }^{3}$ we used TPPAg(II) as a model compound of polyAOTPPAg(II). However, this compound is not considered suitable for the model compound of polyAOTPPAg(II), because $\mathrm{C}=\mathrm{O}$ groups seem to play an important role for the occurrence of the antiferromagnetic interaction. ${ }^{3}$ Thus, it is necessary to measure the magnetic behavior of POTPPAg(II) instead of TPPAg(II) as a model compound. The temperature dependence of magnetic susceptibility of POTPPAg(II) is shown in Figure 1. The $\chi_{M}$ of POTPPAg(II) obeys the Curie-Weiss law with $\theta=-25 \mathrm{~K}$,

Table I. Gram magnetic susceptibility and effective magnetic moment at room temperature

\begin{tabular}{lcc}
\hline Sample & $\begin{array}{c}\chi_{\mathrm{g}} \\
\left(\times 10^{-6} \text { cgsemu }\right)\end{array}$ & $\begin{array}{c}\mu_{\text {eff }} \\
(\mathrm{BM})\end{array}$ \\
\hline TPPAg(II) & 1.14 & 1.79 \\
POTPPAg(II) & 1.13 & 1.78 \\
POLYAOTPPAg(II) & 0.31 & 1.49 \\
POLYMAOTPPAg(II) & 0.92 & 1.72 \\
\hline
\end{tabular}

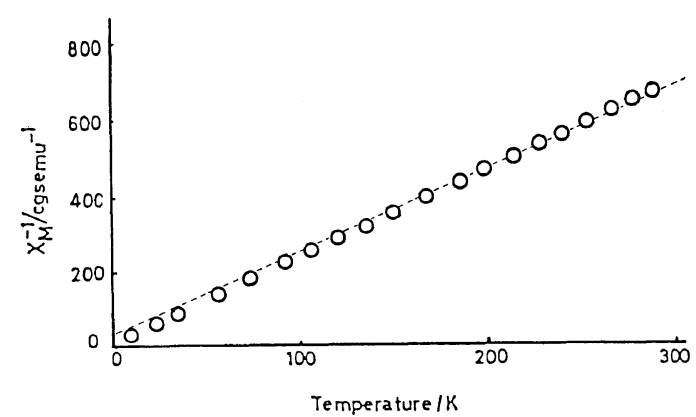

Figure 1. Temperature dependence of magnetic susceptibility of POTPPAg(II). being similar to that of TPPAg(II). This shows that there is only a weak anti-ferromagnetic interaction between POTPPAg(II), although the interaction is a little larger in POTPPAg(II) than TPPAg(II). POTPPAg(II) also has carbonyl group, but the occurrence of the magnetic interaction in POTPPAg(II) was similar to that in TPPAg(II), being much weaker than that in polyAOPPAg(II). These results lead to the conclusion that polymer chains play important roles for magnetic properties.

The temperature dependence of the magnetic susceptibility of polyMAOTPPAg(II) is shown in Figure 2 along with that of polyAOTPPAg(II). Since polyMAOTPPAg(II) as well as polyAOTPPAg(II) have the same kind of TPPAg(II) moieties in the side chains, the magnetic behavior of both polymers was expected to be similar, but was actually quite different. In contrast to strong antiferromagnetism of polyAOTPPAg(II), the $\chi_{M}$ of polyMAOTPPAg(II) seemed to obey the Curie law. Although some deviation from a straight line was observed in the temperature range between 150 and $180 \mathrm{~K}$, extraporation from higher temperature range obeys

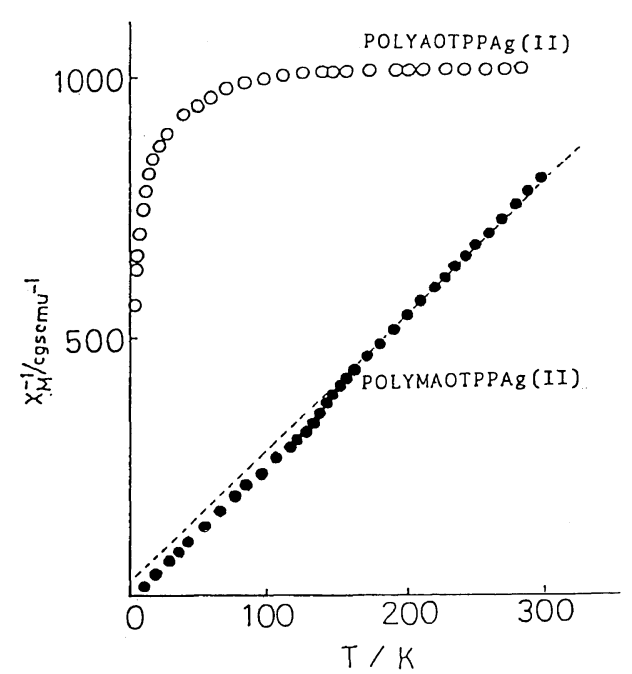

Figure 2. Temperature dependence of magnetic susceptibility of polyAOTPPAg(II) and polyMAOTPPAg(II). 
the Curie-Weiss law with $\theta=-30 \mathrm{~K}$. This shows that there is only a weak antiferromagnetic interaction between TPPAg(II) moieties in the polymer. By molecular orbital calculation, Yamaguchi et al. ${ }^{12}$ showed that the superexchange interaction remarkably depends on the relative location of paramagnetic species. Since we previously found that electric conductivity of polyAOTPP was two order larger than that of polyMAOTPP, ${ }^{9}$ the difference in the temperature dependence of $\chi_{M}$ between both polymers is possibly caused by variation of the relative orientation between TPPAg(II) moieties by methyl groups bound to the main chain.

For an understanding of the effect of the distance between porphyrin moieties on the magnetic behavior, copolymers of AOTPP$\mathrm{Ag}(\mathrm{II})$ and MMA were prepared, and the temperature dependence of their magnetic susceptibility was measured. The results are shown in Figure 3. For poly[AOTPPAg(II)-co-MMA] with $\operatorname{AOTPPAg}(\mathrm{II}): \mathrm{MMA}=1: 0.8$, the $\chi_{\mathrm{M}}$ deviated from the Curie law, although the deviation is much smaller than that of polyAOTPPAg(II). Extraporation from data in a higher temperature region seems to obey the Curie-Weiss law with $\theta=-150 \mathrm{~K}$, suggesting that there is relatively strong antiferromagnetic interaction between TPPAg(II) moieties in the copolymer. For poly[AOTPPAg(II)co-MMA] with AOTPPAg(II): $\mathrm{MMA}=1: 2.6$, the $\chi_{M}$ obeys the Curie-Weiss law with $\theta=$

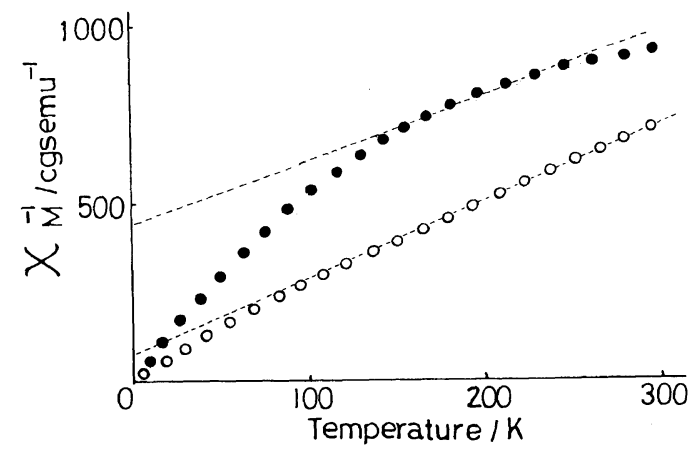

Figure 3. Temperature dependence of magnetic susceptibility of polyAOTPPAg(II)-co-MMA. polyAOTPPAg(II)-co-MMA $[1: 0.8] ; \bigcirc \bigcirc \bigcirc$, polyAOTPPAg(II)-co-MMA $[1: 2.6]$.



Figure 4. ESR spectra of POTPPAg(II), TPPAg(II), and polyAOTPPAg(II) (solid powder, at room temperature. - - POTPPAg(II);--.--, polyAOTPPAg(II); -----, TPPAg(II). 
$-30 \mathrm{~K}$, suggesting that there is weak antiferromagnetic interaction between TPPAg(II) moieties in the side chains. Thus the antiferromagnetic interaction between TPPAg(II) moieties in the side chains of these copolymers is remarkably influenced by changing the average distance of TPPAg(II) moieties.

\section{ESR Spectra of Porphinatosilver(II) Complex}

The ESR spectra of POTPPAg(II) and polyAOTPPAg(II) are shown in Figure 4 along with of TPPAg(II). Anisotropic $g$ factors $\left(g_{\|}\right.$and $\left.g_{\perp}\right)$ were observed for TPPAg(II) and POTPPAg(II). But the line-shape of the latter became a little closer to the symmetrical pattern than that of the former, suggesting that the exchange interaction of POTPPAg(II) is bigger than that of TPPAg(II). On the other hand, a symmetrical line of an isotropic $g$ factor was observed in the case of polyAOTPPAg(II), indicating that an exchange of electron spins through the side chains in the polymer is much larger than those in the model compounds, and consequently, that the magnetic field on any electron is apparently isotropic in polyAOTPPAg(II). ${ }^{3}$

The ESR spectra of poly[AOTPPAg(II)-coMMA] are shown in Figure 5. At first sight, the ESR spectra of poly[AOTPPAg(II)-co-
MMA] seem to show a symmetrical pattern, but in fact, the maximum peak-heights of signals were different from the minimum peakheights in both spectra. In the case of the copolymer with AOTPPAg(II): $M M A=1: 2.6$, the ratio of the peak-height of maximum to minimum was larger than that of the copolymer [AOTPPAg(II): MMA $=1: 0.8]$. These spectra show that, $g_{\|}$and $g_{\perp}$ were not perfectly averaged. Accordingly, we conclude that exchange interaction is weaker in the copolymer, in which the content of TPPAg(II) is smaller.

The ESR spectrum of polyMAOTPPAg(II) is shown in Figure 6 along with that of polyAOTPPAg(II). The former also seems to be similar to the latter, although the former was broader than the latter. But the intensity of maximum of the signal was distinct from that of minimum peak, indicating that $g_{\|}$and $g_{\perp}$ also were not perfectly averaged. This finding shows that the exchange interaction of polyMAOTPPAg(II) was weaker than that of polyAOTPPAg(II).

ESR spectra qualitatively show that the exchange interaction decreases in the order:

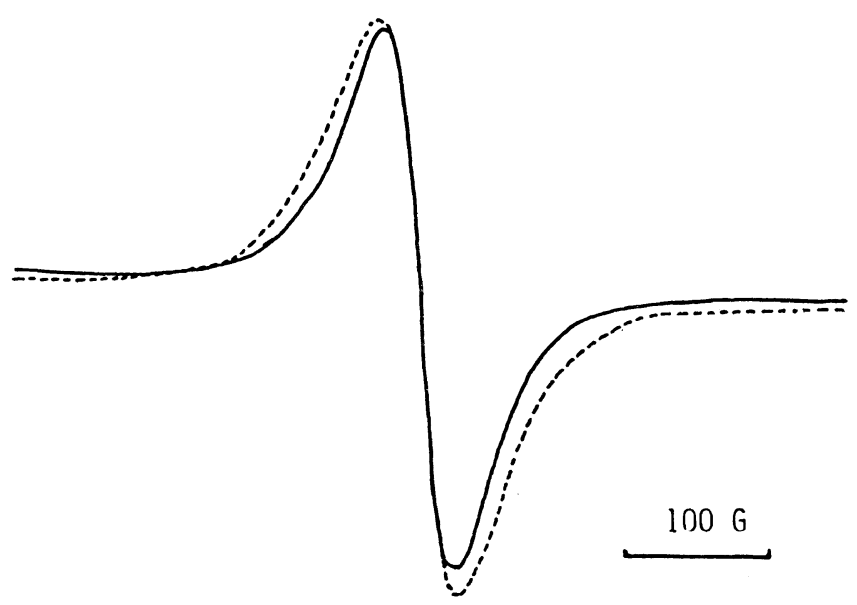

Figure 5. ESR spectra of polyAOTPPAg(II)-co-MMA (solid powder, at room temperature). --..--, polyAOTPPAg(II)-co-MMA $(1: 2.6) ; \_$, polyAOTPPAg(II)-co-MMA $(1: 0.8)$. 


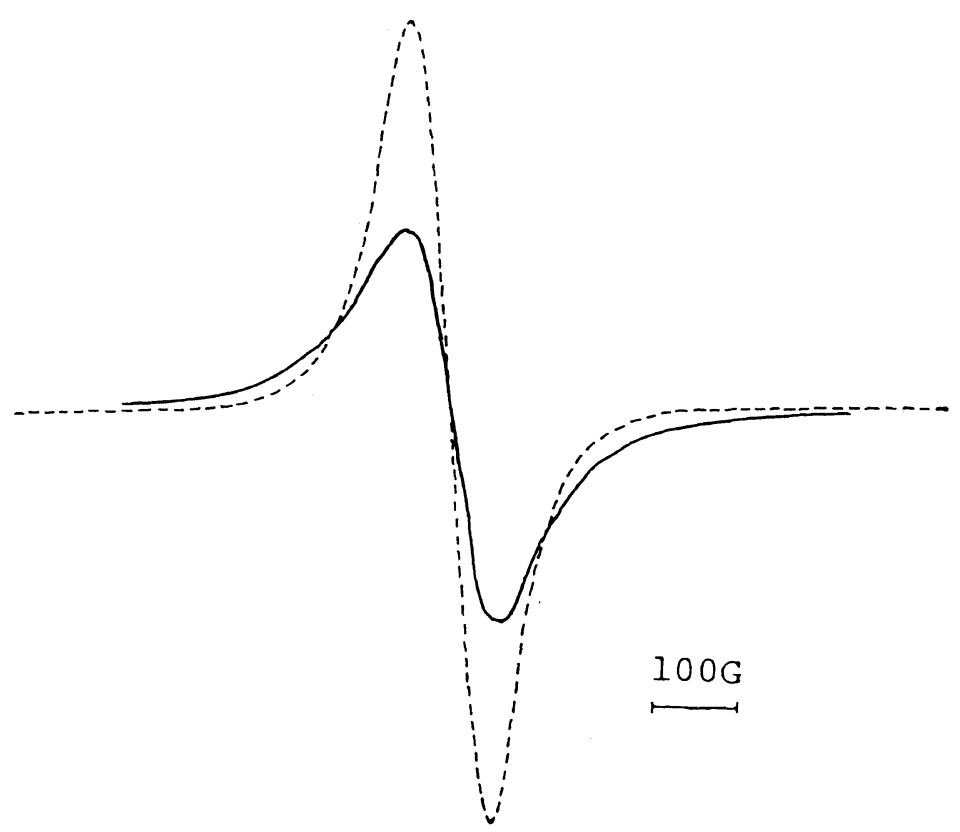

Figure 6. ESR spectra of polyMAOTPPAg(II) and polyAOTPPAg(II) (solid powder, at room temperature). - - , polyMAOTPPAg(II); -----, polyAOTPPAg(II).

\section{PolyAOTPPAg(II)}

$>$ Poly[AOTPPAg(II)-co-MMA] $(1: 0.8)$

$>$ Poly[AOTPPAg(II)-co-MMA] $(1: 2.6)$

$=$ PolyMAOTPPAg(II) $>$ POTPPAg(II)

$>$ TPPAg(II)

This order in the exchange interaction is in good agreement with the results of measurements of magnetic susceptibility.

Accordingly, antiferromagnetic interaction of polyAOTPPAg(II) can be reasonably explained in terms of superexchange interaction, which is remarkably influenced by the distance between paramagnetic species, and by the orientation of paramagnetic species.

Comparison of the magnetic susceptibility and ESR spectrum of polyAOTPPAg(II) with those of POTPPAg(II) reveals that polyAOTPPAg(II) has much stronger antiferromagnetic interactions between TPPAg(II) moieties than POTPPAg(II), which indicates that a polymer chain plays an important role for the occurrence of magnetic interactions. Com- parison of the magnetic properties of polyAOTPPAg(II) with that of polyMAOTPP$\mathrm{Ag}(\mathrm{II})$ shows that the relative location of TPP$\mathrm{Ag}(\mathrm{II})$ moieties is an important factor for the magnetic ordering. Moreover, increase in the distance between TPPAg(II) moieties due to MMA units in copolymers decreased magnetic interaction. These findings are consistent with the previous concept that antiferromagnetic exchange interaction in polyAOTPPAg(II) is possibly caused by the superexchange interaction through the $\mathrm{C}=\mathrm{O}$ group between $\mathrm{Ag}(\mathrm{II})$ ions.

Acknowledgements. The authors are very grateful to Emeritus Professor Shun-ichi Nozakura, Osaka University, for his valuable comments and encouragement. This work was partly supported by a Grant-in-Aid for Scientific Research on the Priority Area "Macromolecule-Complexes (No. 62612001)" from the Ministry of Education, Science, and Culture of Japan. 


\section{REFERENCES}

1. A. A. Ovchinkov, Dokl. Acad. Nauk SSSR., 236, 928 (1978).

2. A. L. Buchachenko, Dokl. Acad. Nauk SSSR., 244, 1146 (1979).

3. M. Kamachi, H. Akimoto, W. Mori, and M. Kishita, Polym. J., 16, 23 (1984).

4. M. Kamachi, M. Tamaki, Y. Morishima, S. Nozakura, W. Mori, and M. Kishita, Polym. J., 14, 363 (1982).

5. M. Kamachi, H. Enomoto, M. Shibasaka, W. Mori, and M. Kishita, Polym. J., 18, 439 (1986).

6. K. M. More, S. S. Eaton, and G. R. Eaton, J. Am.
Chem. Soc., 103, 1087 (1981).

7. R. G. Little, J. A. Anton, P. A. Loach, and S. A. Ibers, J. Heterocycl. Chem., 12, 343 (1975).

8. M. Kamachi, H. Akimoto, and S. Nozakura, J. Polym. Sci., Polym. Lett. Ed., 21, 693 (1983).

9. M. Kamachi, X. S. Cheng, T. Kida, A. Kajiwara, M. Shibasaka, and S. Nagata, Macromolecules, 20, 2665 (1987).

10. G. D. Dorough, J. R. Miller, and F. M. Heunnekens, J. Am. Chem. Soc., 73, 4315 (1951).

11. A. Earnshaw, "Introduction to Magnetochemistry," Academic Press, London, 1968, p 213.

12. K. Yamaguchi, Kinouzairyo, 7, 47 (1986). 